\title{
First-principles studies of $\mathrm{SnS}_{2}, \mathrm{MoS}_{2}$ and $\mathrm{WS}_{2}$ stacked van der Waals hetero-multilayers
}

\author{
H. B. Mabiala-Poaty ${ }^{\mathrm{a}, \mathrm{b}}$, D. H. Douma ${ }^{\mathrm{a}, \mathrm{b}}$, B. R. Malonda-Boungou ${ }^{\mathrm{a}}$, R. E. \\ Mapashac $^{c}$, B. M'Passi-Mabiala ${ }^{a, b}$ \\ ${ }^{a}$ Groupe de Simulations Numériques en Magnétisme et Catalyse (GSMC), Faculté des \\ Sciences et Techniques, Université Marien Ngouabi, BP 69 Brazzaville, Congo. \\ ${ }^{b}$ Unité de Recherche en Nanomatériaux et Nanotechnologies, Institut National de Recherche \\ en Sciences Exactes et Naturelles (IRSEN), Brazzaville, Congo. \\ ${ }^{c}$ Department of Physics, University of Pretoria, Pretoria 0002, South Africa.
}

\begin{abstract}
We present the energetics, structural and electronic properties of $\mathrm{SnS}_{2}$ monolayer stacked with $\mathrm{MoS}_{2}$ and $\mathrm{WS}_{2}$ monolayers making the van der Waals heterolayers using the first-principles methods. The exchange-correlation functionals used are the LDA, GGA functionals as well as the newly developed variants of non local van der Waals (vdW) exchange-correlation functionals, namely vdW-DF-revPBE and vdW-DF2-C09. We also considered the combinations of hetero-layers that involve all the three $\mathrm{SnS}_{2}, \mathrm{MoS}_{2}$ and $\mathrm{WS}_{2}$ stacked together. All the investigated hetero-layers have a short decay (offset) of the equilibrium lattice parameters compared to the $\mathrm{SnS}_{2}$ single layer one. Except for the GGAPBE functional, all the functionals predict the interlayer distances closer to the previous theoretical and experimental studies. The hetero-layers that have relative low binding energies are indirect band gap semiconductors, while those with dramatically high binding energies are weakly or strongly metallic. This study gave another avenue of altering the energetics and electronic properties of $\mathrm{SnS}_{2}$ monolayer through vertical stacking with $\mathrm{MoS}_{2}$ and $\mathrm{WS}_{2}$. The variation in band gap enables these newly predicted hetero-layers to be suitable candidates for designing novel devices for nanoelectronic and optoelectronic technology, which includes energy storage, photodetectors, thermophotovoltaic.
\end{abstract}

Keywords: $\mathrm{SnS}_{2}, \mathrm{MoS}_{2}, \mathrm{WS}_{2}$, van der Waals hetero-layers, Electronic properties, van der Waals exchange-correlation functionals, first-principles 
methods.

\section{Introduction}

After the experimental synthesis of the free standing graphene in 2004 [1, 2], the other two dimensional (2D) materials such as hexagonal boron nitride (h$\mathrm{BN}$ ), transition metal dichalcogenides (TMDs) [3] such as $\mathrm{MoS}_{2}, \mathrm{MoSe}_{2}, \mathrm{WS}_{2}$, $\mathrm{WSe}_{2}$, etc gained a lot of attention. Amongst of these, the TMDs [3] have become attractive due to their potential practical applications [4, 5] in electronic and opto-electronic devices. These can be possible due to unique physical characters such as indirect or direct large band gaps, reduced dimensionality and great mechanical strength in the TMDs systems $\underline{6}$.

The 2D TMDs materials are layered systems, with the transition metals sandwiched between the chalcogen elements covalently bonded together. For few layered TMDs systems, the bonding along the vertical axis or out of plane is due to the van der Waals interactions (vdW) [7, 8. The bulk TMDs hexagonal phases present the indirect band gap, whereas they are direct in monolayer phases $[8,9$. Because of the unique crystal structure, high anisotropy and perculiar electronic properties [10, 11, 12, the 2D TMDs become one of the most studied materials in the fundamental research and technological applications. These materials are easily synthesizable through different methods including chemical vapor deposition, chemical, mechanical and electrochemical exfoliation, and are stable at room temperature [10, 11, 13, 14, 15, 16, 17.

Although the TMDs are relatively large band gap systems, the band gap controllability is the major requirement in the nanotechnology applications. To achieve this, modifying the electronic properties of TMDs continues unabated using different methods such as reducing dimensions, intercalation, alloying, creating heterostructures, etc [18. Recently, the most popular or promising method is the stacking of layers along the vertical axis (vertical heterolayers). This method is practically feasible both experimentally and theoretically [19, 20. 21. He et al. 9] investigated the stacking effects on the electronic and 
optical properties of bilayer TMDs, namely $\mathrm{MoS}_{2}, \mathrm{MoSe}_{2}, \mathrm{WS}_{2}$ and $\mathrm{WSe}_{2}$. It has been established that there is a transition from direct-to-indirect band gap going from monolayer to bilayer. It was concluded that the stacked hetero-layers is another promising possibility that can tune the electronic properties of the main material system. Terrones et al. [22] demonstrated that a direct band gap ranging from $0.79 \mathrm{eV}$ to $1.16 \mathrm{eV}$ can be achieved in TMDs hetero-bilayers with change in stacking.

The Sn-dichalcogenides have a TMDs-like structure. Recently, the theoretical prediction [23, 24] and experimental synthesis of the $\mathrm{SnS}_{2}$ monolayers [5, 25, 26, has attracted considerable attention for its promising chances of being exploited in various electronic devices [13, 23, 27, 28, 29, 30. $\mathrm{SnS}_{2}$ is an indirect band gap semiconductor over the entire thickness range from bulk to single-layer [24]. With the band gap of about $2.2 \mathrm{eV}, \mathrm{SnS}_{2}$ showed the attractive visible light photocatalytic activity which makes it the promising materials in photoelectronic devices and solar cells [31, 32. It displayes interesting charge carrier mobility, mechanical and thermophysical properties, useful in design and the development of new materials [24, 33. Controlling the electronic properties of $\mathrm{SnS}_{2}$ through stacking, in particular involving the homo and hetero-layers has been considered lately [34, 35. In our previous work we have shown, using Density Functional Theory (DFT) calculations, that the stability and electronic properties of the $\mathrm{SnS}_{2}$ multilayers depend on the stacking sequences 36. All the $\mathrm{SnS}_{2}$ multilayer configurations was found to be stable and band gaps tuned with the increase in the number of stacked layers. This study can be extended in the hetero-layer of $\mathrm{SnS}_{2}$ with others TMDs. Zhang et al. 35. have successfully synthesized $\mathrm{SnS}_{2}-\mathrm{MoS}_{2}, \mathrm{SnS}_{2}-\mathrm{WS}_{2}$ and $\mathrm{WS}_{2}-\mathrm{SnS}_{2}$ van der Waals hetero-bilayers using a chemical vapor deposition method at mild temperatures. Theoretical investigations on the electronic properties of these hetero-bilayers was also done [35, and the results confirmed the experiments in altering the electronic properties of the pristine $\mathrm{SnS}_{2}$ monolayer. The creation of multilayers further alters the electronic properties of the main monolayer structure [35, 37, 38, and could be used to understand the transition from direct to 
indirect band gap observed in the hetero-multilayer systems. The hetero-trilayer of $\mathrm{SnS}_{2}$ stacked with $\mathrm{MoS}_{2}$ monolayers should also be plausible, and it will be interesting to examine the band gap variation with the number of hetero-layers. It is interesting to compare the results of standard LDA and GGA functionals [39] with those of the newly developed variants of non-local van der Waals exchange correlation functionals, i.e. vdW-DF-revPBE [40] and vdW-DF2-C09 [41, 42 in such systems. These vdW exchange correlation functionals are known to correctly capture the effects of vdW forces in complex systems, and they are efficient in describing a wide range of systems without too much computational cost [43. The vdW-DF-revPBE functional generally predicts relatively weak binding energy in the case of stacked nanosheets, but it severely overestimates the interlayer distances [40, 44]. To solve this overestimation, the vdW-DF2C09 functional has been used, since it has been proved to be suitable for such systems [41, 42, 44].

In the present work, we studied the interlayer interaction energy, structural (in-plane and out of plane) and electronic properties of $\mathrm{SnS}_{2}$ stacked with $\mathrm{MoS}_{2}$ and $\mathrm{WS}_{2}$ nanosheets making van der Waals hetero-bilayers and trilayers using various DFT methods. We considered in more details the hetero-layers that involves the stacking of $\mathrm{SnS}_{2}$ stacked with $\mathrm{MoS}_{2}$ and $\mathrm{WS}_{2}$ nanosheets and compare with previous works on the similar systems. Our results suggest that the newly studied hetero-multilayers can be an effective way for controlling the band gap of $\mathrm{SnS}_{2}$ nanosheets to meet the requirements of electronic applications at favourable conditions.

This paper is organized as follow: in section II, the details of our computational methods are described. The results of our DFT calculations for $\mathrm{SnS}_{2}$, $\mathrm{MoS}_{2}$ and $\mathrm{WS}_{2}$ sheets and $\mathrm{SnS}_{2}$ heterostructures are given in Section II. The conclusion and perspectives are presented in the final section. 


\section{Computational method}

Our first-principles calculations have been performed using QUANTUM ESPRESSO distribution [45] package which uses a plane wave basis set within the pseudopotential approach and periodic boundary conditions. We use the well-known LDA and GGA exchange-correlation functionals, and the newly developed two variants of non-local van der Waals exchange correlation functionals, namely vdW-DF-revPBE and vdW-DF2-C09 which take into account the vdW forces [40, 41, 42]. The Kohn-Sham (KS) orbitals and the charge density are represented using basis sets consisting of the plane waves up to a maximal kinetic energy of $35 \mathrm{Ry}$ and $350 \mathrm{Ry}$, respectively. The Brillouin zone is sampled using the Monkhorst-Pack scheme [46] with a grid of $7 \times 7 \times 1$ and $10 \times 10 \times 1$ k-points mesh for GGA and LDA functionals respectively. We have used the supercells consisting of $1 \times 1$ unit cell, containing 3, 6 and 9 atoms for one, two and three layers respectively. The layers are separated from their periodic images by at least $15 \AA$ of vacuum in order to minimized their image interactions. The parameters of $10^{-7}$ Ry is set for convergence on the total energy in self consistent cycle. The in-plane lattice parameter input is set to the $3.70 \AA$ (the lattice parameter of $\mathrm{SnS}_{2}$ from GGA functional). All coordinates and lattice constants are allowed to relax. Our preliminary results including spin-orbit interactions have shown no major differences compared to those calculated without spin-orbit interactions. Both the band structures with and without spin-orbit interactions possess nearly equivalent bands spliting at the VBM (valence band maximum) in agreement with [4]. Indeed, the contribution of spin-orbit interaction were excluded in this work.

\section{Results and Discussion}

In this work, all the calculations were performed on the most stable phase of $\mathrm{SnS}_{2}, \mathrm{MoS}_{2}$ and $\mathrm{WS}_{2}$ [8, 25, 48, showing a layered $\mathrm{CdI}_{2}$-type structure in which the metallic atom is sandwiched between two $\mathrm{S}$ atoms in a hexagonal closed packed lattice shown in Figure 1. Using the GGA-PBE functional 
only, we performed the structural optimization of $\mathrm{SnS}_{2}, \mathrm{MoS}_{2}$ and $\mathrm{WS}_{2}$ isolated monolayers to calculate the equilibrium in-plane lattice constants, and thereafter their band structures were plotted to examine the electronic properties. Table 1 summarizes the results, and compares them with the available experimental data and previous theoretical results using GGA-PBE functional, and the obtained lattice parameters of $3.70 \AA\left(\mathrm{SnS}_{2}\right), 3.17 \AA\left(\mathrm{MoS}_{2}\right)$ and $3.15 \AA$ $\left(\mathrm{WS}_{2}\right)$ are in agreement with these previous works as shown in this table. The calculated in-plane M-S bondlengths are in good agreement with the literature (see table 1). Comparing the lattice constants of these isolated layers, it is noted that the lattice constants of $\mathrm{MoS}_{2}$ and $\mathrm{WS}_{2}$ are very close to each other suggesting that if these layers can be stacked on top of each other there should be no issue of lattice mismatch, and this could be the reason of the excessive literature $[22,49,50,51,52,53,54,55,56,57$. However, there is notable lattice constants difference between $\mathrm{SnS}_{2}$ and $\mathrm{MoS}_{2}$ of $0.53 \AA$. This suggests that the stacking of $\mathrm{SnS}_{2}$ layer with $\mathrm{MoS}_{2}$ layer $\left(\mathrm{SnS}_{2} / \mathrm{MoS}_{2}\right)$ vertically would have a lattice mismatch and will not be easily achieved ideally, therefore a special care should be made in the construction of the atomic models. In the experimental study of the synthesis of $\mathrm{SnS}_{2} / \mathrm{MoS}_{2}$ and $\mathrm{SnS}_{2} / \mathrm{WS}_{2}$ stacked layers, it was mentioned that there is lattice mismatch of about $15 \%$ along the $\vec{a}$ direction, importantly tolerated by the van der Waals epitaxy [35. When modeling these type of hetero-layers, it is imperative to use the exchange correlation functionals that can take into account the effects of van der Waals forces.

In Figure 2, we present the calculated band structures of $\mathrm{SnS}_{2}, \mathrm{MoS}_{2}$ and $\mathrm{WS}_{2}$ isolated sheets. The $\mathrm{MoS}_{2}$ and $\mathrm{WS}_{2}$ sheets are direct band gap semiconductors with the valence band maximum (VBM) and the conduction band minimum (CBM) located at the K-high symmetry point, in agreement with the previous studies $\left[8,49,23,58,59,60\right.$. The $\mathrm{SnS}_{2}$ inherently is an indirect band gap with the CBM located at the M-point, while the VBM is seen between the $\Gamma$-point and M-point . Table 1 shows the band gap of $1.57 \mathrm{eV}, 1.59 \mathrm{eV}$ and $1.75 \mathrm{eV}$ for $\mathrm{SnS}_{2}, \mathrm{MoS}_{2}$ and $\mathrm{WS}_{2}$ isolated sheets respectively, in agreement with the previous DFT studies [8, 49, 23, 58, 59, 60. Comparing with the experi- 
ments [25, 56, 61, 62, our GGA-PBE results show a slight underestimation as expected. For the stacking of $\mathrm{SnS}_{2}$ with $\mathrm{MoS}_{2}$ or $\mathrm{SnS}_{2}$ with $\mathrm{WS}_{2}$, it will be interesting to see how their CBM and VBM rearrange and the band gaps change. To investigate which electron orbital states contributes for VBM and CBM, we present the total and partial density of states (Figure 3). It is noted that the VBM and CBM of $\mathrm{SnS}_{2}$ monolayer are mainly contributed by $p$-S orbital states, while those of $\mathrm{MoS}_{2}$ and $\mathrm{WS}_{2}$ are mainly contributed by $d$ states from Mo and W.

(a)

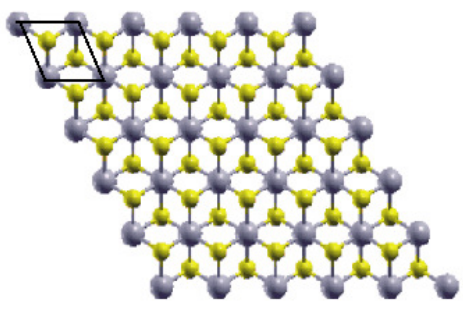

(b)

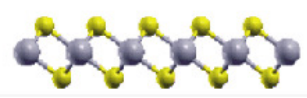

(c)

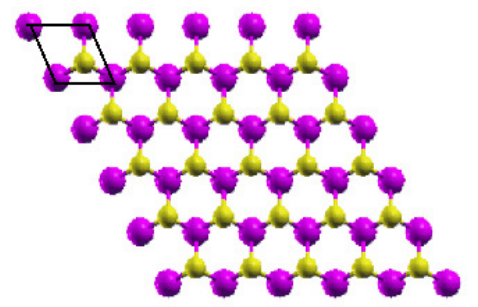

(d)

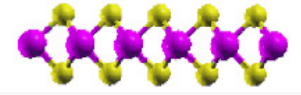

Figure 1 (Color online) The top (a) and side (b) views of $5 \times 5 \times 1 \mathrm{SnS}_{2}$ isolated sheet. The top (c) and side (d) views of $5 \times 5 \times 1 \mathrm{MoS}_{2}\left(\right.$ resp. $\left.\mathrm{WS}_{2}\right)$ isolated sheet. The Sn, Mo (resp. W), and S are represented by the gray, purple and yellow spheres respectively. All the calculations were performed on a unit cell delimited by the black lines in ( $\mathrm{a}$ and $\mathrm{c}$ ). The $5 \times 5 \times 1$ supercell size is used to clearly show the hexagons of the heterostructures. 
(a)

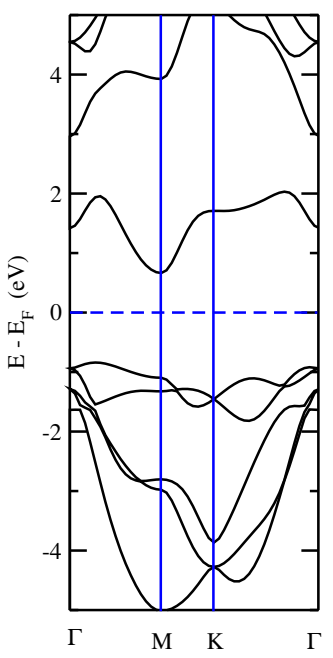

(b)

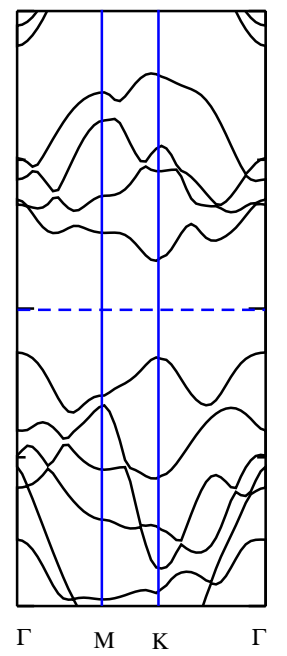

(c)

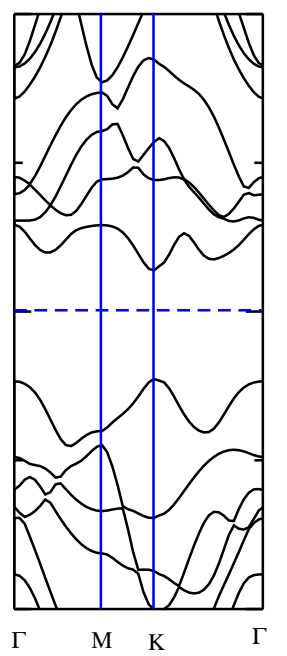

Figure 2 (Color online) The calculated band structure plots of (a) the $\mathrm{SnS}_{2}$, (b) $\mathrm{MoS}_{2}$ and (c) $\mathrm{WS}_{2}$ isolated layers obtained using the GGA-PBE functional. The Fermi level is set to $0.00 \mathrm{eV}$. 
(a)

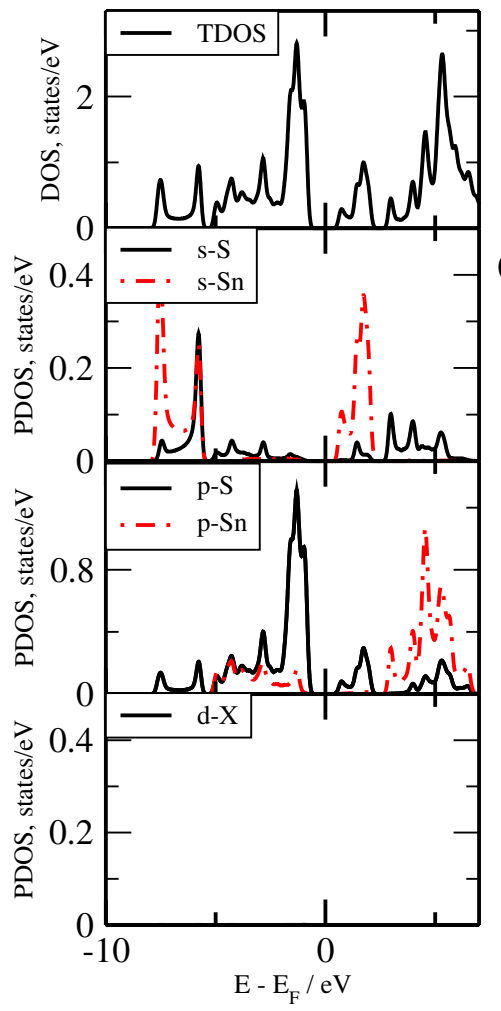

(b)

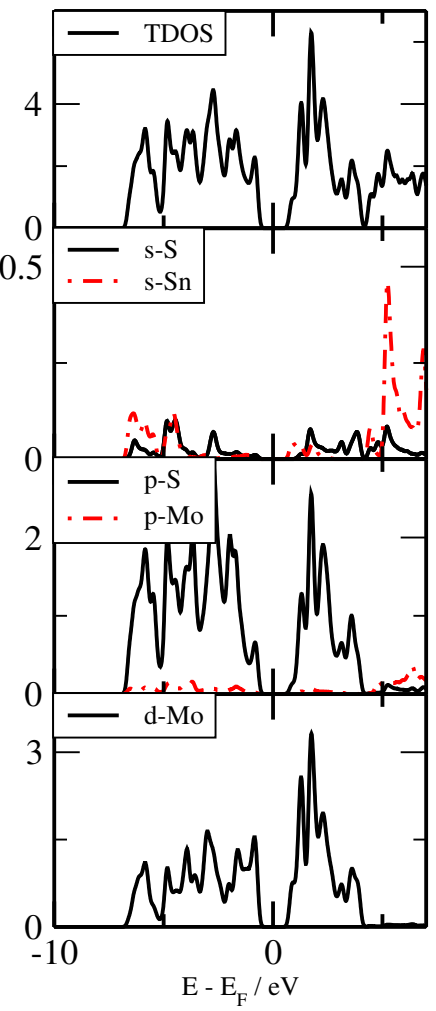

(c)

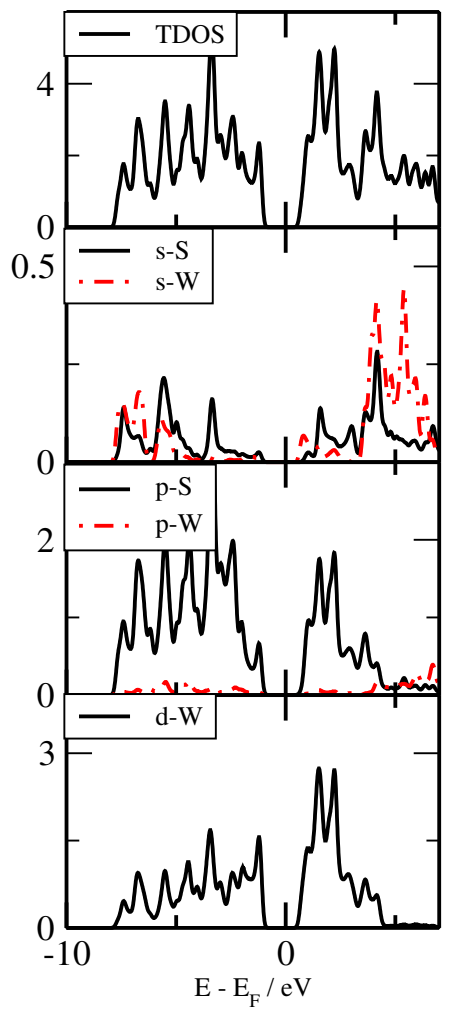

Figure 3 (Color online) The calculated total and partial DOS of (a) $\mathrm{SnS}_{2}$, (b) $\mathrm{MoS}_{2}$ and (c) $\mathrm{WS}_{2}$ isolated layer systems. The Fermi level is set to $0.00 \mathrm{eV}$.

\section{1. $M S_{2} / S n S_{2}(M=M o, W)$ hetero-bilayer}

Firstly, we examined the properties of $\mathrm{WS}_{2} / \mathrm{SnS}_{2}$ and $\mathrm{MoS}_{2} / \mathrm{SnS}_{2}$ stacked hetero-bilayers, using the LDA, GGA-PBE, vdW-DF-revPBE and vdW-DF2C09 functionals. For both systems, the following stacking sequence was considered; the Sn atoms are directly facing the $\mathrm{W}$ or Mo atoms on the adjacent layer, while the $\mathrm{S}$ atoms on the top layer directly face the $\mathrm{S}$ atoms on the bottom as shown in Figure 4(a). This isolated stacking layers is similar structurally and comparable in energy to the bulk phases of $\mathrm{MoS}_{2}$ and $\mathrm{WS}_{2}$ studied by Terrones et al $[22$. 
Table 1: The calculated lattice constants a $(\AA)$, bond lengths $\mathrm{b}_{M-S}(M=S n, M o$ and $W)$ $(\AA)$ and the energy band gaps $\mathrm{E}_{g}(\mathrm{eV})$ of the $\mathrm{SnS}_{2}, \mathrm{MoS}_{2}$ and $\mathrm{WS}_{2}$ isolated sheets. These values are compared with the previous DFT studies as well as the available experimental data. The $(*)$ means the value is not available.

\begin{tabular}{lcccc}
\hline Structures & & $\mathrm{a}_{0}(\AA)$ & $\mathrm{b}_{M-S}(\AA)$ & $\mathrm{E}_{g}(\mathrm{eV})$ \\
\hline $\mathrm{SnS}_{2}$ & This work & 3.70 & 2.60 & 1.57 \\
& Ref & $3.70[23]$ & $2.60[23]$ & $1.57[23]$ \\
& Exp & $3.65[25]$ & $2.65[25]$ & $2.23[62]$ \\
\hline $\mathrm{WS}_{2}$ & This work & 3.15 & 2.38 & 1.75 \\
& Ref & $3.19[59]$ & $2.43[49]$ & $1.9[60]$ \\
& Exp & $3.15[61]$ & $*$ & $2.1[56]$ \\
\hline $\mathrm{MoS}_{2}$ & This work & 3.17 & 2.37 & 1.59 \\
& Ref & $3.16[8]$ & $2.41[49]$ & $1.60[58]$ \\
& Exp & $3.15[8]$ & $2.38[8]$ & $1.8[56]$ \\
\hline
\end{tabular}

After full geometry optimization, the in-plane lattice constants $\mathrm{a}_{0}$, out of plane interlayer spacings $\mathrm{d}_{0}$, interlayer binding energies $\mathrm{E}_{b}$ (as the energy needed to keep the stacked layer coupled) and the electronic properties were calculated.

The binding energies $\mathrm{E}_{b}$ are defined as:

$$
E_{\mathrm{b}}=E_{\text {layers }}-i E_{S n S_{2}}-j E_{M o S_{2}}-k E_{W S_{2}}
$$

in which $E_{\text {layers }}, E_{S n S_{2}}, E_{M o S_{2}}$ and $E_{W S_{2}}$ are the energy of the stacked heterolayers, the energy of a $S n S_{2}, M o S_{2}$ and $W S_{2}$ single layer respectively. Where $\mathrm{i}=1,2$ and $\mathrm{j}, \mathrm{k}=0,1,2$, refering to the number of $S n S_{2}, M o S_{2}$ and $W S_{2}$ layers respectively. According to equation (1), a negative value of $E_{\mathrm{b}}$ corresponds to a strongly coupled hetero-layer, while a positive value corresponds to weakly coupled layers.

From GGA-PBE functionals, we found that for both $\mathrm{WS}_{2} / \mathrm{SnS}_{2}$ and $\mathrm{MoS}_{2} / \mathrm{SnS}_{2}$ hetero-bilayers, the value of $\mathrm{a}_{0}$ is offset compared to the $\mathrm{SnS}_{2}$ single layer one (see tables 1 and 21. Quantitatively, the in-plane lattice parameter $\mathrm{a}_{0}$ of $\mathrm{WS}_{2} / \mathrm{SnS}_{2}$ 
(resp. $\mathrm{MoS}_{2} / \mathrm{SnS}_{2}$ ) hetero-layer is $0.32 \AA$ (resp. $0.37 \AA$ ) larger than the $\mathrm{SnS}_{2}$ single layer one, while it is $0.23 \AA$ (resp. $0.21 \AA$ ) lower than the $\mathrm{WS}_{2}\left(\right.$ resp. $\mathrm{MoS}_{2}$ ) single layer one. This lattice offset is also noted in M-S in-plane bondlengths. Excepted for the vdW-DF-revPBE functional, all other functionals agree with the experimental values in ref [35].

From table 2 all the considered functionals show that the $\mathrm{MoS}_{2} / \mathrm{SnS}_{2}$ stacked hetero-bilayer has larger $\mathrm{d}_{0}$ and lower $\mathrm{E}_{b}$ than $\mathrm{WS}_{2} / \mathrm{SnS}_{2}$. Apart from the GGAPBE, all other functionals predict $\mathrm{d}_{0}$ relatively closer to the experimental and theoretical values [9, 35, 51. Although, the values of $\mathrm{E}_{b}$ are not negative, this could be due to the lattice mismatch between $\mathrm{SnS}_{2}$ and $\mathrm{WS}_{2}$ (resp. $\mathrm{MoS}_{2}$ ), observed experimentally by Zhang et al [35. The small variations in these functionals in predicting the $\mathrm{E}_{b}$ is an indication that the van der Waals interactions are possible but in the very small magnitudes. Although, the $\mathrm{MoS}_{2} / \mathrm{SnS}_{2}$ bilayer has larger $\mathrm{d}_{0}$ than $\mathrm{WS}_{2} / \mathrm{SnS}_{2}$, its lower $\mathrm{E}_{b}$ could suggests that there is great dipole-dipole interactions between Sn and Mo than between Sn and W.

Figure 5 shows the band structures of (a) $\mathrm{MoS}_{2} / \mathrm{SnS}_{2}$ and (b) $\mathrm{WS}_{2} / \mathrm{SnS}_{2}$ hetero-bilayers predicted by all the functionals considered. The plots reveal that the creation of $\mathrm{MoS}_{2} / \mathrm{SnS}_{2}$ or $\mathrm{WS}_{2} / \mathrm{SnS}_{2}$ hetero-bilayer rearrange the VBM and $\mathrm{CBM}$ of $\mathrm{MoS}_{2}, \mathrm{SnS}_{2}$ and $\mathrm{WS}_{2}$ layers, resulting to the indirect band gap semiconductors with the VBM at the $\Gamma$-point and CBM located at the K-point. By comparing tables 1 and 2, we observed that the stacking of $\mathrm{MoS}_{2} / \mathrm{SnS}_{2}$ and $\mathrm{WS}_{2} / \mathrm{SnS}_{2}$ layers generally reduces the band gaps of the isolated layers, and this for all the functionals used. This reduction in band gap is mainly contributed by the additional $d$-states coming from Mo (Figure 6(a)) and W (Figure 6(b)) at the VBM. Although the heterostructures are fully relaxed, the noted lattice mismatch could also be the possibility for the band gap reduction. The results presented in this work are for the $1 \times 1$ unit cells. For improved results, a large unit cell could be considered in order to reduce the lattice mismatch effects occured when forming the heterostructures from isolated layers that have significant disparity in the lattice parameters $\left(7 \times 7\right.$ unit cell for $\mathrm{MoS}_{2}\left(\right.$ resp. $\left.\mathrm{WS}_{2}\right)$ and $6 \times 6$ unit cell for $\mathrm{SnS}_{2}$ ). 
Table 2: The calculated physical properties of the $\mathrm{SnS}_{2}$ monolayer stacked with $\mathrm{MoS}_{2}$ and $\mathrm{WS}_{2}$ monolayers obtained using different exchange correlation functionals. The properties calculated are the lattice parameter $a_{0}$, bondlengths $\mathrm{b}_{S n-S}$ and $\mathrm{b}_{W-S}$, interlayer spacing $\mathrm{d}_{0}$, binding energy $\mathrm{E}_{b}$ and band-gap $\mathrm{E}_{g}$. In the case of three stacked layers, there are two values of $\mathrm{d}_{0}$ separated by slash.

\begin{tabular}{|c|c|c|c|c|c|}
\hline Stacking & & GGA-PBE & $\mathrm{PZ}(\mathrm{LDA})$ & vdW-DF-revPBE & vdW-DF2-C09 \\
\hline \multirow[t]{5}{*}{$\mathrm{WS}_{2} / \mathrm{SnS}_{2}$} & $\mathrm{a}_{0}(\AA)$ & 3.38 & 3.29 & 3.45 & 3.35 \\
\hline & $\mathrm{b}_{S n-S / W-S}(\AA)$ & $2.51 / 2.45$ & $2.48 / 2.39$ & $2.54 / 2.48$ & $2.50 / 2.43$ \\
\hline & $\mathrm{d}_{0}(\AA)$ & 6.77 & 5.94 & 6.57 & 5.98 \\
\hline & $\mathrm{E}_{b}(\mathrm{eV} /$ Cell $)$ & 0.882 & 0.865 & 0.677 & 1.338 \\
\hline & $\mathrm{E}_{g}(\mathrm{eV})$ & 0.56 & 0.40 & 0.41 & 0.58 \\
\hline \multirow[t]{5}{*}{$\mathrm{MoS}_{2} / \mathrm{SnS}_{2}$} & $\mathrm{a}_{0}(\AA)$ & 3.39 & 3.29 & 3.45 & 3.31 \\
\hline & $\mathrm{b}_{S n-S / M o-S}(\AA)$ & $2.51 / 2.45$ & $2.47 / 2.40$ & $2.54 / 2.49$ & $2.49 / 2.41$ \\
\hline & $\mathrm{d}_{0}(\AA)$ & 7.22 & 6.54 & 6.88 & 6.55 \\
\hline & $\mathrm{E}_{b}(\mathrm{eV} /$ Cell $)$ & 0.822 & 0.753 & 0.640 & 0.997 \\
\hline & $\mathrm{E}_{g}(\mathrm{eV})$ & 0.32 & 0.53 & 0.26 & 0.47 \\
\hline \multirow[t]{5}{*}{$\mathrm{SnS}_{2} / \mathrm{WS}_{2} / \mathrm{SnS}_{2}$} & $\mathrm{a}_{0}(\AA)$ & 3.48 & 3.22 & 3.54 & 3.26 \\
\hline & $\mathrm{b}_{S n-S / W-S}(\AA)$ & $2.53 / 2.48$ & $2.50 / 2.43$ & $2.58 / 2.52$ & $2.54 / 2.46$ \\
\hline & $\mathrm{d}_{0}(\AA)$ & $7.28 / 6.81$ & $5.74 / 6.50$ & $6.34 / 6.80$ & $5.96 / 6.69$ \\
\hline & $\mathrm{E}_{b}(\mathrm{eV} / \mathrm{Cell})$ & 1.146 & 1.097 & 0.804 & 0.855 \\
\hline & $\mathrm{E}_{g}(\mathrm{eV})$ & & & & \\
\hline \multirow[t]{5}{*}{$\mathrm{SnS}_{2} / \mathrm{MoS}_{2} / \mathrm{SnS}_{2}$} & $\mathrm{a}_{0}(\AA)$ & 3.49 & 3.40 & 3.55 & 3.45 \\
\hline & $\mathrm{b}_{S n-S / M o-S}(\AA)$ & $2.56 / 2.48$ & $2.49 / 2.44$ & $2.57 / 2.52$ & $2.54 / 2.47$ \\
\hline & $\mathrm{d}_{0}(\AA)$ & $6.87 / 6.88$ & $6.35 / 6.24$ & $6.81 / 6.38$ & $6.61 / 5.96$ \\
\hline & $\mathrm{E}_{b}(\mathrm{eV} /$ Cell $)$ & 1.063 & 0.899 & 0.678 & 0.779 \\
\hline & $\mathrm{E}_{g}(\mathrm{eV})$ & & & & \\
\hline \multirow[t]{5}{*}{$\mathrm{WS}_{2} / \mathrm{SnS}_{2} / \mathrm{WS}_{2}$} & $\mathrm{a}_{0}(\AA)$ & 3.31 & 3.21 & 3.36 & 3.27 \\
\hline & $\mathrm{b}_{S n-S / W-S}(\AA)$ & $2.47 / 2.42$ & $2.46 / 2.37$ & $2.51 / 2.46$ & $2.48 / 2.41$ \\
\hline & $\mathrm{d}_{0}(\AA)$ & $7.15 / 6.33$ & $6.61 / 6.02$ & $6.80 / 6.53$ & $6.61 / 6.08$ \\
\hline & $\mathrm{E}_{b}(\mathrm{eV} /$ Cell $)$ & 3.199 & 1.060 & 2.541 & 2.903 \\
\hline & $\mathrm{E}_{g}(\mathrm{eV})$ & & & 0.63 & \\
\hline \multirow[t]{5}{*}{$\mathrm{MoS}_{2} / \mathrm{SnS}_{2} / \mathrm{MoS}_{2}$} & $\mathrm{a}_{0}(\AA)$ & 3.30 & 3.21 & 3.38 & 3.26 \\
\hline & $\mathrm{b}_{S n-S / M o-S}(\AA)$ & $2.49 / 2.42$ & $2.44 / 2.38$ & $2.53 / 2.46$ & $2.50 / 2.41$ \\
\hline & $\mathrm{d}_{0}(\AA)$ & $6.60 / 7.01$ & $6.30 / 6.20$ & $6.21 / 6.73$ & $6.12 / 6.53$ \\
\hline & $\mathrm{E}_{b}(\mathrm{eV} / \mathrm{Cell})$ & 1.094 & 0.918 & 0.732 & 0.810 \\
\hline & $\mathrm{E}_{g}(\mathrm{eV})$ & 0.25 & 0.42 & 0.48 & 0.39 \\
\hline \multirow[t]{5}{*}{$\mathrm{MoS}_{2} / \mathrm{WS}_{2} / \mathrm{SnS}_{2}$} & $\mathrm{a}_{0}(\AA)$ & 3.31 & 3.22 & 3.37 & 3.26 \\
\hline & $\mathrm{b}_{S n-S / W-S / M o-S}(\AA)$ & $2.48 / 2.42 / 2.42$ & $2.44 / 2.37 / 2.38$ & $2.51 / 2.45 / 2.46$ & $2.47 / 2.41 / 2.41$ \\
\hline & $\mathrm{d}_{0}(\AA)$ & $6.32 / 6.28$ & $6.31 / 6.26$ & $6.32 / 6.29$ & $6.31 / 6.26$ \\
\hline & $\mathrm{E}_{b}(\mathrm{eV} / \mathrm{Cell})$ & 1.161 & 1.206 & 0.977 & 1.074 \\
\hline & $\mathrm{E}_{g}(\mathrm{eV})$ & 0.29 & 0.54 & 0.21 & 0.46 \\
\hline \multirow[t]{5}{*}{$\mathrm{WS}_{2} / \mathrm{MoS}_{2} / \mathrm{SnS}_{2}$} & $\mathrm{a}_{0}(\AA)$ & 3.31 & 3.21 & 3.36 & 3.27 \\
\hline & $\mathrm{b}_{S n-S / W-S / M o-S}(\AA)$ & $2.51 / 2.42 / 2.41$ & $2.44 / 2.36 / 2.38$ & $2.53 / 2.45 / 2.45$ & $2.49 / 2.41 / 2.41$ \\
\hline & $\mathrm{d}_{0}(\AA)$ & $7.52 / 6.34$ & $6.34 / 6.24$ & $6.98 / 6.46$ & $6.61 / 6.04$ \\
\hline & $\mathrm{E}_{b}(\mathrm{eV} / \mathrm{Cell})$ & 1.160 & 1.207 & 0.975 & 1.075 \\
\hline & $\mathrm{E}_{g}(\mathrm{eV})$ & 0.32 & 0.43 & 0.30 & 0.37 \\
\hline
\end{tabular}


(a)

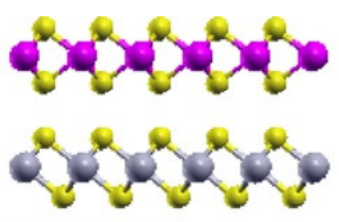

(b)

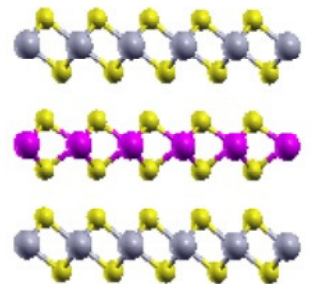

(c)

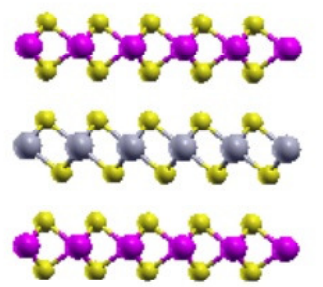

(d)

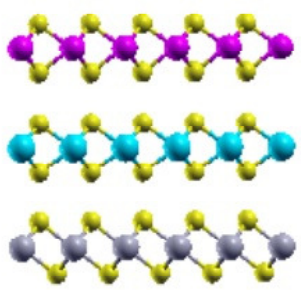

Figure 4 (Color online) The crystal structures of bilayer heterostructure (a) $\mathrm{Mo}(\mathrm{W}) \mathrm{S}_{2} / \mathrm{SnS}_{2}$. The trilayer heterostructures (b) $\mathrm{SnS}_{2} / \mathrm{Mo}(\mathrm{W}) \mathrm{S}_{2} / \mathrm{SnS}_{2}$, (c) $\mathrm{Mo}(\mathrm{W}) \mathrm{S}_{2} / \mathrm{SnS}_{2} / \mathrm{Mo}(\mathrm{W}) \mathrm{S}_{2}$ and (d) $(\mathrm{W}) \mathrm{MoS}_{2} /(\mathrm{Mo}) \mathrm{WS}_{2} / \mathrm{SnS}_{2}$. The light green and purple spheres represent the Mo or $\mathrm{W}$ atoms.
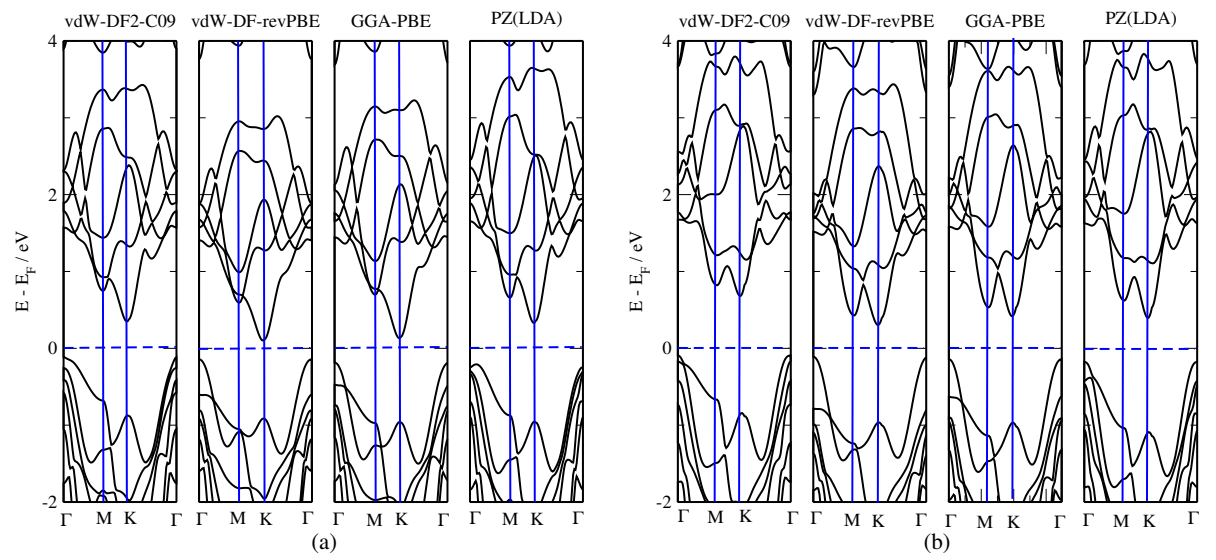

Figure 5 (Color online) The calculated band structure plots of (a) $\mathrm{MoS}_{2} / \mathrm{SnS}_{2}$ and (b) $\mathrm{WS}_{2} / \mathrm{SnS}_{2}$ heterostructures obtained using the four different functionals. The Fermi energy is set to $0.00 \mathrm{eV}$. 
(a)

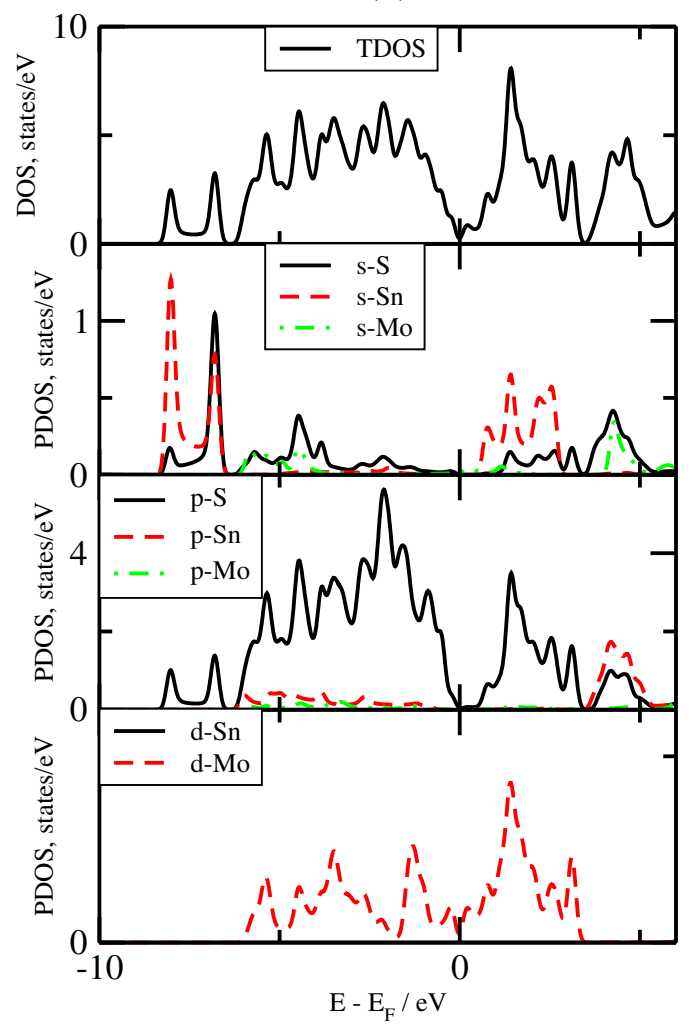

(b)

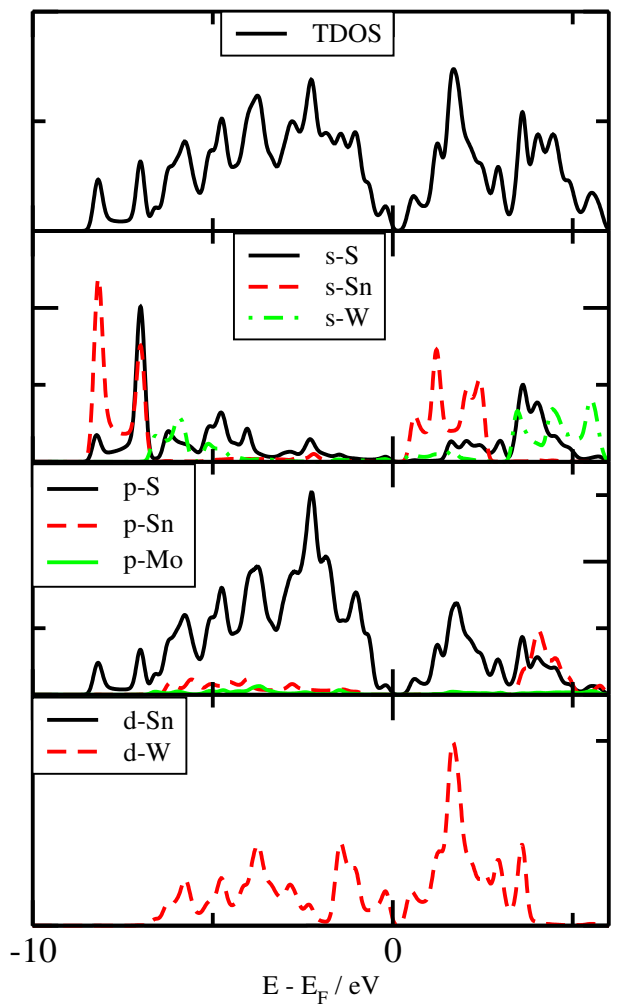

Figure 6 (Color online) The calculated total and partial DOS of (a) $\mathrm{MoS}_{2} / \mathrm{SnS}_{2}$ and (b) $\mathrm{WS}_{2} / \mathrm{SnS}_{2}$ systems. The Fermi level is set to $0.00 \mathrm{eV}$.

\section{2. $S n S_{2} / M S_{2} / S n S_{2}(M=M o, W)$ heterostructures}

The hybrid heterostructures consisting of $\mathrm{MS}_{2}$ sandwiched between two $\mathrm{SnS}_{2}$ monolayers $\left(\mathrm{SnS}_{2} / \mathrm{MoS}_{2} / \mathrm{SnS}_{2}\right.$ and $\mathrm{SnS}_{2} / \mathrm{WS}_{2} / \mathrm{SnS}_{2}$ hetero-trilayers) were also studied in this present work (see Figure 4(b)). Similar hybrid study was earlier performed in the case of graphene or Boron nitride sandwiched between $\mathrm{MoS}_{2}$ layers or between $\mathrm{WS}_{2}$ layers 63 , 64. Table 2 summarizes the physical properties of the $\mathrm{SnS}_{2} / \mathrm{MoS}_{2} / \mathrm{SnS}_{2}$ and $\mathrm{SnS}_{2} / \mathrm{WS}_{2} / \mathrm{SnS}_{2}$ hetero-trilayers. The values of $\mathrm{a}_{0}$ are close to those found in the case of hetero-bilayers. As reported in table 2 the Sn-S, Mo-S and W-S bond lengths calculated using all the functionals are approximatively the same for $\mathrm{SnS}_{2} / \mathrm{MoS}_{2} / \mathrm{SnS}_{2}$ as well as for $\mathrm{SnS}_{2} / \mathrm{WS}_{2} / \mathrm{SnS}_{2}$ 
systems. The calculated $\mathrm{d}_{0}$ for $\mathrm{SnS}_{2} / \mathrm{WS}_{2} / \mathrm{SnS}_{2}$ hetero-trilayers are shorter between the top layer and the second layer $(5.74 \AA, 6.34 \AA$ and 5.96 for LDA, vdW-DF-revPBE and vdW-DF2-C09 functionals respectively) than between the second layer and most bottom layer $(6.50 \AA, 6.80 \AA$ and $6.69 \AA$ for LDA, vdWDF-revPBE and vdW-DF2-C09 functionals respectively) as presented in table 2. Different scenario is noted in the case of $\mathrm{SnS}_{2} / \mathrm{MoS}_{2} / \mathrm{SnS}_{2}$ where the values of $\mathrm{d}_{0}$ are almost equal in both interlayer separations. In the two systems, the lowest $\mathrm{E}_{b}$ is obtained using the vdW-DF-revPBE functional (0.678 eV/Cell and $0.804 \mathrm{eV} /$ Cell for $\mathrm{SnS}_{2} / \mathrm{MoS}_{2} / \mathrm{SnS}_{2}$ and $\mathrm{SnS}_{2} / \mathrm{WS}_{2} / \mathrm{SnS}_{2}$ respectively), suggesting that $\mathrm{MoS}_{2}$ sheet favourably adhere to $\mathrm{SnS}_{2}$ than $\mathrm{WS}_{2}$ sheet.

The band structures related to $\mathrm{SnS}_{2} / \mathrm{MoS}_{2} / \mathrm{SnS}_{2}$ and $\mathrm{SnS}_{2} / \mathrm{WS}_{2} / \mathrm{SnS}_{2}$ heterotrilayers presented in Figure 7, show that an addition of the third layer $\left(\mathrm{SnS}_{2}\right.$ layer) to the hetero-bilayer $\left(\mathrm{SnS}_{2} / \mathrm{MoS}_{2}\right.$ and $\left.\mathrm{SnS}_{2} / \mathrm{WS}_{2}\right)$ closes the band gap. For both structures, the VBM and CBM touch the Fermi level at the $\Gamma$-Point and the K-point respectively, suggesting a weak metallic character which can be easily altered by external factors such as temperature and pressure. In order to understand the origin of closing the band gap, we calculated the partial density of states (PDOS) for $s, p$ and $d$ orbitals of all the atoms in $\mathrm{SnS}_{2} / \mathrm{MoS}_{2} / \mathrm{SnS}_{2}$ and $\mathrm{SnS}_{2} / \mathrm{WS}_{2} / \mathrm{SnS}_{2}$ hetero-layers, and the results are presented in Figures 8(a) and $8(\mathrm{~b})$. In these Figures, one can see that for the two heterostructures, the VBM and CBM at the vicinity of the Fermi level are mainly contributed by the $d$-W and $d$-Mo orbitals. 

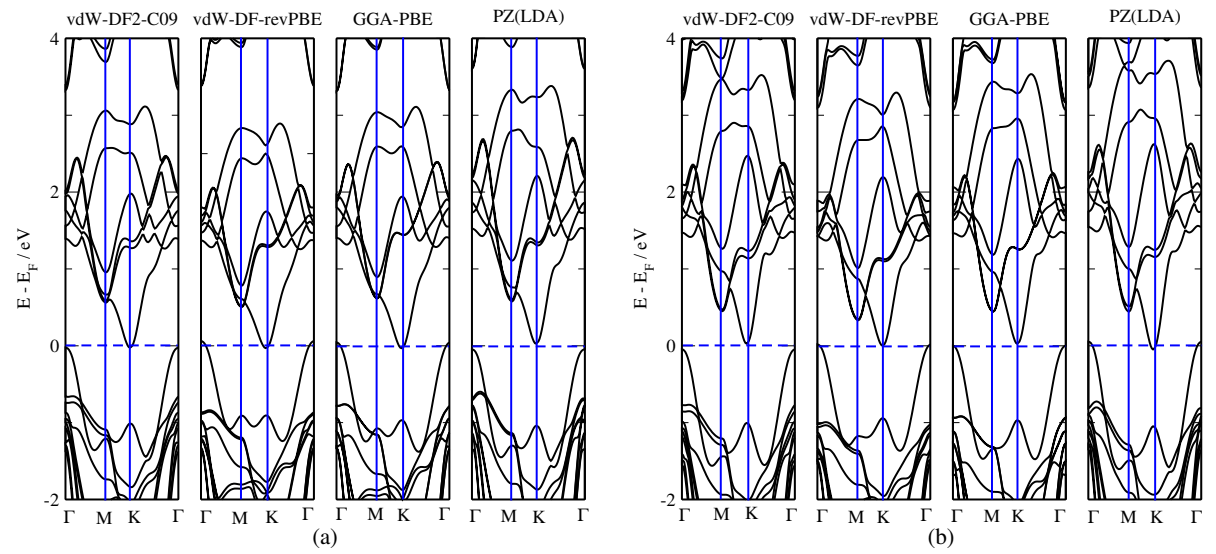

Figure 7 (Color online) The calculated band structure plots of (a) $\mathrm{SnS}_{2} / \mathrm{MoS}_{2} / \mathrm{SnS}_{2}$ and (b) $\mathrm{SnS}_{2} / \mathrm{WS}_{2} / \mathrm{SnS}_{2}$ heterostructures calculated using the four different functionals. The Fermi energy is set to $0.00 \mathrm{eV}$. 
(a)

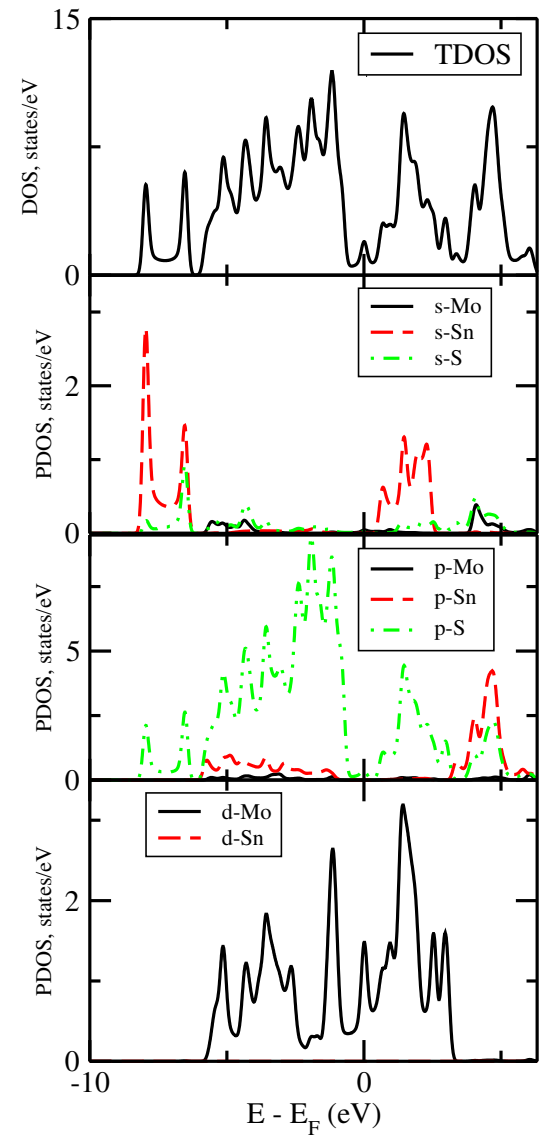

(b)

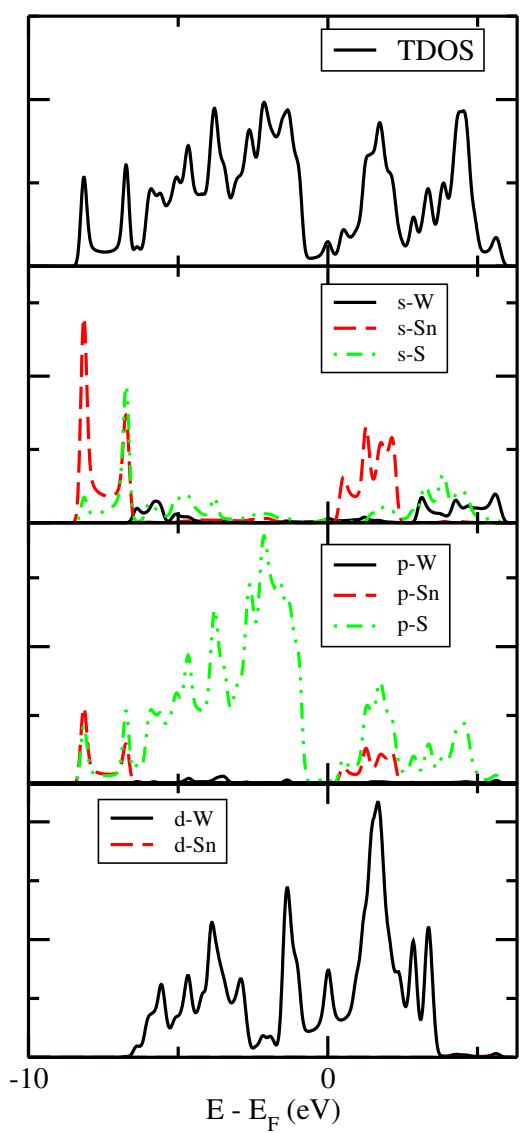

Figure 8 (Color online) The calculated total and partial DOS of (a) $\mathrm{SnS}_{2} / \mathrm{MoS}_{2} / \mathrm{SnS}_{2}$ and (b) $\mathrm{S}_{2} / \mathrm{WS}_{2} / \mathrm{SnS}_{2}$ systems. The Fermi level is set to $0.00 \mathrm{eV}$.

\section{3. $M S_{2} / S n S_{2} / M S_{2}(M=M o, W)$ heterostructures}

The second hybrid hetero-trilayers studied involved $\mathrm{MoS}_{2}$ or $\mathrm{WS}_{2}$ layers sandwiching the $\mathrm{SnS}_{2}$ layer $\left(\mathrm{MoS}_{2} / \mathrm{SnS}_{2} / \mathrm{MoS}_{2}\right.$ and $\left.\mathrm{WS}_{2} / \mathrm{SnS}_{2} / \mathrm{WS}_{2}\right)$ along the vertical axis as shown in Figure 4(c). The calculated in-plane lattice constant $\mathrm{a}_{0}$ are similar in the two systems, for each exchange-correlation functional (see table 2 . These are the lowest lattice constant compared to the previous heterostructures $\left(\mathrm{Mo}(\mathrm{W}) \mathrm{S}_{2} / \mathrm{SnS}_{2}\right.$ and $\left.\mathrm{SnS}_{2} / \mathrm{Mo}(\mathrm{W}) \mathrm{S}_{2} / \mathrm{SnS}_{2}\right)$. This is the result of the dominance of $\mathrm{MoS}_{2}$ or $\mathrm{WS}_{2}$ layers in the hybrid systems. In fact, during 
the geometry optimization of the unit cell, the relatively short bondlength $b_{W-S}$ $\left(\right.$ resp. $\left.b_{M o-S}\right)$ in $\mathrm{WS}_{2}\left(\right.$ resp. $\mathrm{MoS}_{2}$ ) layer reduces the $b_{S n-S}$ in $\mathrm{SnS}_{2}$ layer, resulting to a shortest $\mathrm{a}_{0}$. Although, the interlayer spacings in $\mathrm{MoS}_{2} / \mathrm{SnS}_{2} / \mathrm{MoS}_{2}$ and $\mathrm{WS}_{2} / \mathrm{SnS}_{2} / \mathrm{WS}_{2}$ hetero-trilayers do not deviate much from the previous hetero-trilayers $\left(\mathrm{Mo}(\mathrm{W}) \mathrm{S}_{2} / \mathrm{SnS}_{2}\right.$ and $\left.\mathrm{SnS}_{2} / \mathrm{Mo}(\mathrm{W}) \mathrm{S}_{2} / \mathrm{SnS}_{2}\right)$, while the $\mathrm{E}_{b}$ value of $\mathrm{WS}_{2} / \mathrm{SnS}_{2} / \mathrm{WS}_{2}$ is dramatically very higher than that of $\mathrm{MoS}_{2} / \mathrm{SnS}_{2} / \mathrm{MoS}_{2}$ as shown in table 2. This suggests that the vertical interaction of Mo with Sn through dipole-dipole is slightly favourable than that of W with Sn, and it could also be more possible to synthesize the $\mathrm{MoS}_{2} / \mathrm{SnS}_{2} / \mathrm{MoS}_{2}$ than the $\mathrm{WS}_{2} / \mathrm{SnS}_{2} / \mathrm{WS}_{2}$ system although endothermically. Experimentally, it was reported that these type of systems can be achieved at mild temperatures 35 .

Figures $9(\mathrm{a}$ and $\mathrm{b})$ show the band structures of the $\mathrm{MoS}_{2} / \mathrm{SnS}_{2} / \mathrm{MoS}_{2}$ and $\mathrm{WS}_{2} / \mathrm{SnS}_{2} / \mathrm{WS}_{2}$ hetero-trilayer systems. Table 2 shows that the lowest binding energy of the $\mathrm{MoS}_{2} / \mathrm{SnS}_{2} / \mathrm{MoS}_{2}$ hetero-trilayer system has a narrow band gap whose the magnitude depends on the functional used. This magnitude is nearly the same for the $\mathrm{MoS}_{2} / \mathrm{SnS}_{2}$ (resp. $\mathrm{WS}_{2} / \mathrm{SnS}_{2}$ ) hetero-bilayer (see table 2). We can further see the metallic feature in the case of $\mathrm{WS}_{2} / \mathrm{SnS}_{2} / \mathrm{WS}_{2}$ system by all the functionals excepted the LDA functional which predicts the semiconducting character with a band gap of $0.63 \mathrm{eV}$.
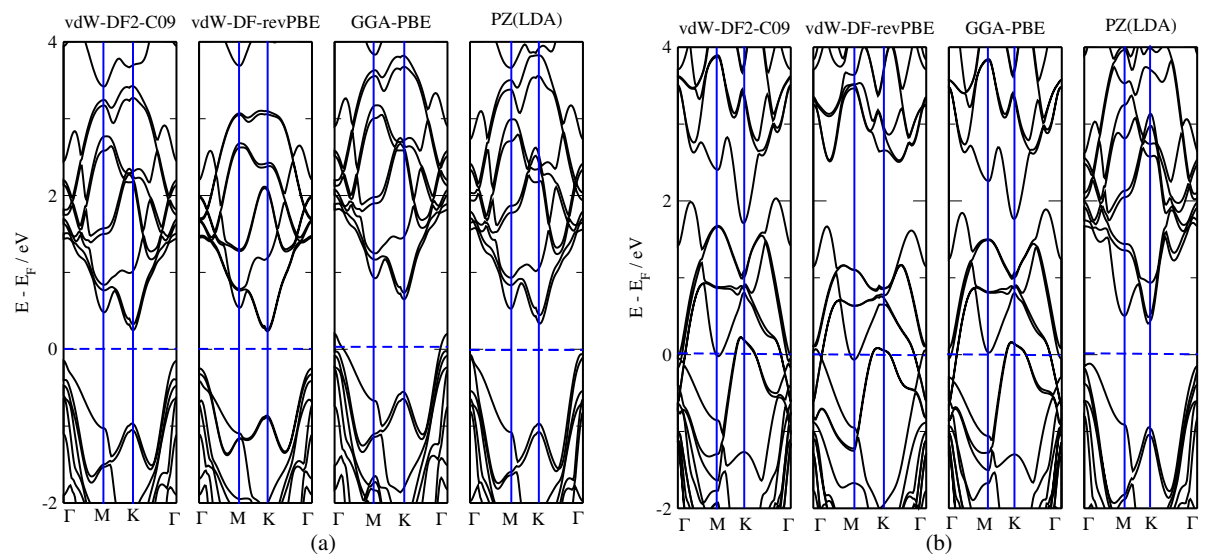

Figure 9 (Color online) The calculated band structures plots of (a) $\mathrm{MoS}_{2} / \mathrm{SnS}_{2} / \mathrm{MoS}_{2}$ and (b) $\mathrm{WS}_{2} / \mathrm{SnS}_{2} / \mathrm{WS}_{2}$ hetero-trilayer obtained using the four different functionals. The 
Fermi energy is set to $0.00 \mathrm{eV}$.

\section{4. $W(M o) S_{2} / M o(W) S_{2} / S n S_{2}$ heterostructures}

Finally, we studied the $\mathrm{MoS}_{2} / \mathrm{WS}_{2} / \mathrm{SnS}_{2}$ and $\mathrm{WS}_{2} / \mathrm{MoS}_{2} / \mathrm{SnS}_{2}$ hetero-trilayer systems which is the combination that was never studied before (see Figure $4(d))$. In these hetero-trilayer systems, the value of $\mathrm{a}_{0}$ does not deviate from the previously studied $\mathrm{MoS}_{2} / \mathrm{SnS}_{2} / \mathrm{MoS}_{2}$ and $\mathrm{WS}_{2} / \mathrm{SnS}_{2} / \mathrm{WS}_{2}$ hetero-trilayer systems as shown in table 2. We notice a slight difference in $\mathrm{Sn}-\mathrm{S}$ bondlength of $\mathrm{MoS}_{2} / \mathrm{WS}_{2} / \mathrm{SnS}_{2}$ compared to $\mathrm{WS}_{2} / \mathrm{MoS}_{2} / \mathrm{SnS}_{2}$ heterostructures while their $\mathrm{E}_{b}$ are almost equal.

The band structures of $\mathrm{WS}_{2} / \mathrm{MoS}_{2} / \mathrm{SnS}_{2}$ and $\mathrm{MoS}_{2} / \mathrm{WS}_{2} / \mathrm{SnS}_{2}$ hetero-trilayer systems are presented in Figures 10 ((a) and (b)). Both systems are narrow indirect band gap semiconductors, with $\mathrm{MoS}_{2} / \mathrm{WS}_{2} / \mathrm{SnS}_{2}$ system having $0.29 \mathrm{eV}$ (GGA), $0.54 \mathrm{eV}(\mathrm{LDA}), 0.21 \mathrm{eV}$ (vdW-DF-revPBE) and $0.46 \mathrm{eV}$ (vdW-DF2C09), and $\mathrm{WS}_{2} / \mathrm{MoS}_{2} / \mathrm{SnS}_{2} 0.32 \mathrm{eV}$ (GGA), $0.43 \mathrm{eV}(\mathrm{LDA}), 0.30 \mathrm{eV}$ (vdW-DFrevPBE) and $0.33 \mathrm{eV}$ (vdW-DF2-C09). This study demonstrated the avenue of altering the physical properties of $\mathrm{SnS}_{2}$ layer through stacking with the $\mathrm{MoS}_{2}$ and $\mathrm{WS}_{2}$ layers.
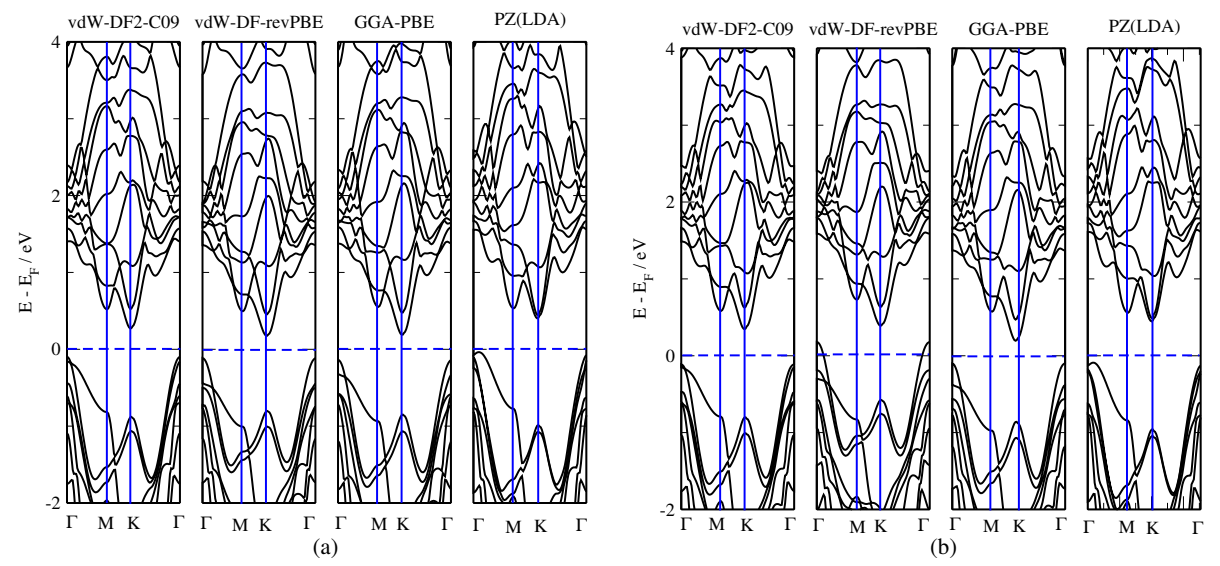

Figure 10 (Color online) The calculated band structure plots of (a) $\mathrm{WS}_{2} / \mathrm{MoS}_{2} / \mathrm{SnS}_{2}$ and (b) $\mathrm{MoS}_{2} / \mathrm{WS}_{2} / \mathrm{SnS}_{2}$ systems obtained using the four different functionals. The Fermi level is set to $0.00 \mathrm{eV}$. 


\section{Conclusion and perspectives}

We have systematically investigated the interlayer interaction, structural and electronic properties of the $\mathrm{SnS}_{2}, \mathrm{MoS}_{2}$ and $\mathrm{WS}_{2}$ stacked hetero-layers using first-principles methods. The hetero-layers consist of two or three stacked layers, particulary $\mathrm{SnS}_{2}$ combined with either $\mathrm{MoS}_{2}$ or $\mathrm{WS}_{2}$. We also considered the new combination that involves all the three $\mathrm{SnS}_{2}, \mathrm{MoS}_{2}$ and $\mathrm{WS}_{2}$ stacked together. All the investigated hetero-layers have a short decay (offset) of the equilibrium lattice parameters compared to the $\mathrm{SnS}_{2}$ single layer one which influences the electronic properties. Apart from the GGA-PBE functional, all other functionals predict the interlayer distances closer to the previous theoretical and experimental studies. The vdW-DF-revPBE functional gives the lowest interlayer binding energies while the GGA-PBE predicts the highest. It was established that the dipole-dipole interactions play a significant role on the energetics of the heterostructures. The hetero-layers that have relative low binding energies are indirect band gap semiconductors, while those of dramatically high binding energies are weakly or strongly metallic. Our newly studied heterolayers are plausible and require further experimental characterization. This study gave another avenue of altering the energetics and electronic properties of $\mathrm{SnS}_{2}$ monolayer through vertical stacking. The variation in band gap enables these newly predicted hetero-layers to be suitable candidates for designing novel devices for nanoelectronic and optoelectronic technology, which includes energy storage, photodetectors, thermophotovoltaic. In this work, only qualitative effect of different exchange-correlation functionals on the band structure has been study. The quantitative effect can be further investigated by using the hybrid DFT-HSE06 functional known as better to describe accurately the electronic band gap.

\section{Acknowledgments}

The calculations were carried out using computational resources through the Ohio Supercomputing Center and the Centre of High Performance Comput- 
ing (CHPC). REM acknowledges University of Pretoria for financial support. The authors are grateful to F. N. Andriambelaza and L. Tchibota-Poaty for constructive suggestions.

\section{References}

[1] K. S. Novoselov, A. K. Geim, S. V. Morozov, D. Jiang, Y. Zhang, S. V. Dubonos, I. V. Grigorieva, A. A. Firsov, Electric field effect in atomically thin carbon films, science 306 (5696) (2004) 666-669.

[2] K. Novoselov, D. Jiang, F. Schedin, T. Booth, V. Khotkevich, S. Morozov, A. Geim, Two-dimensional atomic crystals, Proceedings of the National Academy of Sciences of the United States of America 102 (30) (2005) 1045110453.

[3] R. Gordon, D. Yang, E. Crozier, D. Jiang, R. Frindt, Structures of exfoliated single layers of ws 2 , mos 2 , and mose 2 in aqueous suspension, Physical Review B 65 (12) (2002) 125407.

[4] C. S. Rout, P. D. Joshi, R. V. Kashid, D. S. Joag, M. A. More, A. J. Simbeck, M. Washington, S. K. Nayak, D. J. Late, Superior field emission properties of layered ws2-rgo nanocomposites, Scientific reports 3.

[5] P. D. Joshi, D. S. Joag, C. S. Rout, D. J. Late, Photosensitive field emission study of sns2 nanosheets, Journal of Vacuum Science \& Technology B, Nanotechnology and Microelectronics: Materials, Processing, Measurement, and Phenomena 33 (3) (2015) 03C106.

[6] Y. Zhang, J. Ye, Y. Matsuhashi, Y. Iwasa, Ambipolar mos2 thin flake transistors, Nano letters 12 (3) (2012) 1136-1140.

[7] K. F. Mak, K. He, C. Lee, G. H. Lee, J. Hone, T. F. Heinz, J. Shan, Tightly bound trions in monolayer mos2, Nature materials 12 (3) (2013) 207-211.

[8] E. S. Kadantsev, P. Hawrylak, Electronic structure of a single mos 2 monolayer, Solid State Communications 152 (10) (2012) 909-913. 
[9] J. He, K. Hummer, C. Franchini, Stacking effects on the electronic and optical properties of bilayer transition metal dichalcogenides mos 2, mose 2, ws 2, and wse 2, Physical Review B 89 (7) (2014) 075409.

[10] Q. H. Wang, K. Kalantar-Zadeh, A. Kis, J. N. Coleman, M. S. Strano, Electronics and optoelectronics of two-dimensional transition metal dichalcogenides, Nature nanotechnology 7 (11) (2012) 699-712.

[11] M. Chhowalla, H. S. Shin, G. Eda, L.-J. Li, K. P. Loh, H. Zhang, The chemistry of two-dimensional layered transition metal dichalcogenide nanosheets, Nature chemistry 5 (4) (2013) 263-275.

[12] D. Kong, H. Wang, J. J. Cha, M. Pasta, K. J. Koski, J. Yao, Y. Cui, Synthesis of mos2 and mose2 films with vertically aligned layers, Nano letters 13 (3) (2013) 1341-1347.

[13] Y. Sun, H. Cheng, S. Gao, Z. Sun, Q. Liu, Q. Liu, F. Lei, T. Yao, J. He, S. Wei, et al., Freestanding tin disulfide single-layers realizing efficient visible-light water splitting, Angewandte Chemie International Edition 51 (35) (2012) 8727-8731.

[14] Y.-H. Lee, X.-Q. Zhang, W. Zhang, M.-T. Chang, C.-T. Lin, K.-D. Chang, Y.-C. Yu, J. T.-W. Wang, C.-S. Chang, L.-J. Li, et al., Synthesis of largearea mos2 atomic layers with chemical vapor deposition, Advanced Materials 24 (17) (2012) 2320-2325.

[15] Y. Shi, W. Zhou, A.-Y. Lu, W. Fang, Y.-H. Lee, A. L. Hsu, S. M. Kim, K. K. Kim, H. Y. Yang, L.-J. Li, et al., van der waals epitaxy of mos2 layers using graphene as growth templates, Nano letters 12 (6) (2012) 2784-2791.

[16] Y. Zhan, Z. Liu, S. Najmaei, P. M. Ajayan, J. Lou, Large-area vapor-phase growth and characterization of mos2 atomic layers on a sio2 substrate, Small 8 (7) (2012) 966-971.

[17] W. Zhu, T. Low, Y.-H. Lee, H. Wang, D. B. Farmer, J. Kong, F. Xia, P. Avouris, Electronic transport and device prospects of monolayer molyb- 
denum disulphide grown by chemical vapour deposition, Nature Communications 5 (2014) 3087.

[18] H. Wang, H. Yuan, S. S. Hong, Y. Li, Y. Cui, Physical and chemical tuning of two-dimensional transition metal dichalcogenides, Chemical Society Reviews 44 (9) (2015) 2664-2680.

[19] H. Zeng, G.-B. Liu, J. Dai, Y. Yan, B. Zhu, R. He, L. Xie, S. Xu, X. Chen, W. Yao, et al., Optical signature of symmetry variations and spin-valley coupling in atomically thin tungsten dichalcogenides, Scientific reports 3 .

[20] S. Srivastava, B. Avasthi, Layer type tungsten dichalcogenide compounds: their preparation, structure, properties and uses, Journal of materials science 20 (11) (1985) 3801-3815.

[21] D. Wickramaratne, F. Zahid, R. K. Lake, Electronic and thermoelectric properties of few-layer transition metal dichalcogenides, The Journal of chemical physics 140 (12) (2014) 124710.

[22] H. Terrones, F. López-Urías, M. Terrones, Novel hetero-layered materials with tunable direct band gaps by sandwiching different metal disulfides and diselenides, Scientific reports 3 (2013) 1549.

[23] H. L. Zhuang, R. G. Hennig, Theoretical perspective of photocatalytic properties of single-layer sns2, Physical Review B 88 (11) (2013) 115314.

[24] Y. Huang, E. Sutter, J. T. Sadowski, M. Cotlet, O. L. Monti, D. A. Racke, M. R. Neupane, D. Wickramaratne, R. K. Lake, B. A. Parkinson, et al., Tin disulfide, an emerging layered metal dichalcogenide semiconductor: Materials properties and device characteristics, ACS nano 8 (10) (2014) 1074310755.

[25] F. Al-Alamy, A. Balchin, M. White, The expansivities and the thermal degradation of some layer compounds, Journal of Materials Science 12 (10) (1977) 2037-2042. 
[26] J.-H. Ahn, M.-J. Lee, H. Heo, J. H. Sung, K. Kim, H. Hwang, M.-H. Jo, Deterministic two-dimensional polymorphism growth of hexagonal n-type sns2 and orthorhombic p-type sns crystals, Nano letters 15 (6) (2015) 37033708.

[27] C. Xia, X. Zhao, Y. Peng, H. Zhang, S. Wei, Y. Jia, First-principles study of group v and vii impurities in sns2, Superlattices and Microstructures 85 (2015) 664-671.

[28] L. Sun, W. Zhou, Y. Liang, L. Liu, P. Wu, Magnetic properties in fe-doped sns2: Density functional calculations, Computational Materials Science 117 (2016) 489-495.

[29] G. Kiruthigaa, C. Manoharan, M. Bououdina, S. Ramalingam, C. Raju, Structural, optical and photocatalytic properties of ce-doped sns2 nanoflakes, Solid State Sciences 44 (2015) 32-38.

[30] L. Sun, W. Zhou, Y. Liu, Y. Lu, Y. Liang, P. Wu, A first-principles study on the origin of magnetism induced by intrinsic defects in monolayer sns2, Computational Materials Science 126 (2017) 52-58.

[31] C. Xia, J. An, T. Wang, S. Wei, Y. Jia, Sn1-xtixs2 ternary alloys: A new visible optical material, Acta Materialia 72 (2014) 223-228.

[32] R. Lucena, F. Fresno, J. C. Conesa, Hydrothermally synthesized nanocrystalline tin disulphide as visible light-active photocatalyst: Spectral response and stability, Applied Catalysis A: General 415 (2012) 111-117.

[33] X. He, H. Shen, Ab initio calculations of band structure and thermophysical properties for sns2 and snse2, Physica B: Condensed Matter 407 (7) (2012) $1146-1152$.

[34] J. Li, J. Shen, Z. Ma, K. Wu, Thickness-controlled electronic structure and thermoelectric performance of ultrathin sns2 nanosheets, Scientific Reports 7 (1) (2017) 8914. 
[35] X. Zhang, F. Meng, J. R. Christianson, C. Arroyo-Torres, M. A. Lukowski, D. Liang, J. R. Schmidt, S. Jin, Vertical heterostructures of layered metal chalcogenides by van der waals epitaxy, Nano Lett 14 (6) (2014) 3047-3054.

[36] H. B. Mabiala-Poaty, D. H. Douma, B. M'Passi-Mabiala, R. E. Mapasha, Structural and electronic properties of sns2 stacked nanosheets: An abinitio study, Journal of Physics and Chemistry of Solids 120 (2018) 211217.

[37] A. A. Puretzky, L. Liang, X. Li, K. Xiao, K. Wang, M. Mahjouri-Samani, L. Basile, J. C. Idrobo, B. G. Sumpter, V. Meunier, et al., Low-frequency raman fingerprints of two-dimensional metal dichalcogenide layer stacking configurations, ACS nano 9 (6) (2015) 6333-6342.

[38] A. Kumar, P. Ahluwalia, Electronic structure of transition metal dichalcogenides monolayers $1 \mathrm{~h}-\mathrm{mx} 2(\mathrm{~m}=\mathrm{mo}, \mathrm{w} ; \mathrm{x}=\mathrm{s}$, se, te $)$ from ab-initio theory: new direct band gap semiconductors, The European Physical Journal B 85 (6) (2012) 186.

[39] J. P. Perdew, A. Zunger, Self-interaction correction to density-functional approximations for many-electron systems, Physical Review B 23 (10) (1981) 5048.

[40] M. Dion, H. Rydberg, E. Schröder, D. C. Langreth, B. I. Lundqvist, Van der waals density functional for general geometries, Physical review letters 92 (24) (2004) 246401.

[41] V. R. Cooper, Van der waals density functional: An appropriate exchange functional, Physical Review B 81 (16) (2010) 161104.

[42] K. Lee, É. D. Murray, L. Kong, B. I. Lundqvist, D. C. Langreth, Higheraccuracy van der waals density functional, Physical Review B 82 (8) (2010) 081101. 
[43] R. E. Mapasha, R. C. Andrew, N. Chetty, Van der waals density-functional study of $100 \%$ hydrogen coverage on bilayer graphene, Computational Materials Science 78 (2013) 1-8.

[44] R. E. Mapasha, A. M. Ukpong, N. Chetty, Ab initio studies of hydrogen adatoms on bilayer graphene, Physical Review B 85 (20) (2012) 205402.

[45] P. Giannozzi, S. Baroni, N. Bonini, M. Calandra, R. Car, C. Cavazzoni, D. Ceresoli, G. L. Chiarotti, M. Cococcioni, I. Dabo, et al., Quantum espresso: a modular and open-source software project for quantum simulations of materials, Journal of physics: Condensed matter 21 (39) (2009) 395502 .

[46] H. J. Monkhorst, J. D. Pack, Special points for brillouin-zone integrations, Physical review B 13 (12) (1976) 5188.

[47] J. M. Gonzalez, I. I. Oleynik, Layer-dependent properties of sns2 and snse2 two-dimensional materials, Physical Review B 94 (12) (2016) 125443.

[48] C. Bacaksiz, S. Cahangirov, A. Rubio, R. T. Senger, F. M. Peeters, H. Sahin, Bilayer sns 2: Tunable stacking sequence by charging and loading pressure, Physical Review B 93 (12) (2016) 125403.

[49] D. Wang, L.-M. Liu, S.-J. Zhao, Z.-Y. Hu, H. Liu, Potential application of metal dichalcogenides double-layered heterostructures as anode materials for li-ion batteries, The Journal of Physical Chemistry C 120 (9) (2016) $4779-4788$.

[50] A. Castellanos-Gomez, E. Cappelluti, R. Roldán, N. Agraït, F. Guinea, G. Rubio-Bollinger, Electric-field screening in atomically thin layers of mos2: the role of interlayer coupling, Advanced materials 25 (6) (2013) 899-903.

[51] H.-P. Komsa, A. V. Krasheninnikov, Electronic structures and optical properties of realistic transition metal dichalcogenide heterostructures from first principles, Physical Review B 88 (8) (2013) 085318. 
[52] K. Kośmider, J. Fernández-Rossier, Electronic properties of the mos 2-ws 2 heterojunction, Physical Review B 87 (7) (2013) 075451.

[53] L. Kou, T. Frauenheim, C. Chen, Nanoscale multilayer transition-metal dichalcogenide heterostructures: band gap modulation by interfacial strain and spontaneous polarization, The journal of physical chemistry letters 4 (10) (2013) 1730-1736.

[54] M. Bernardi, M. Palummo, J. C. Grossman, Extraordinary sunlight absorption and one nanometer thick photovoltaics using two-dimensional monolayer materials, Nano letters 13 (8) (2013) 3664-3670.

[55] L.-Y. Gan, Y.-J. Zhao, D. Huang, U. Schwingenschlögl, First-principles analysis of mos $2 /$ ti 2 c and mos $2 /$ ti 2 c y 2 ( $\mathrm{y}=\mathrm{f}$ and oh) all-2d semiconductor/metal contacts, Physical Review B 87 (24) (2013) 245307.

[56] H. Wang, F. Liu, W. Fu, Z. Fang, W. Zhou, Z. Liu, Two-dimensional heterostructures: fabrication, characterization, and application, Nanoscale 6 (21) (2014) 12250-12272.

[57] S. Tongay, W. Fan, J. Kang, J. Park, U. Koldemir, J. Suh, D. S. Narang, K. Liu, J. Ji, J. Li, et al., Tuning interlayer coupling in large-area heterostructures with cvd-grown mos2 and ws2 monolayers, Nano letters 14 (6) (2014) 3185-3190.

[58] N. Lu, H. Guo, L. Wang, X. Wu, X. C. Zeng, van der waals trilayers and superlattices: modification of electronic structures of mos2 by intercalation, Nanoscale 6 (9) (2014) 4566-4571.

[59] C. Gong, H. Zhang, W. Wang, L. Colombo, R. M. Wallace, K. Cho, Band alignment of two-dimensional transition metal dichalcogenides: Application in tunnel field effect transistors, Applied Physics Letters 103 (5) (2013) 053513 . 
[60] T. Georgiou, H. Yang, R. Jalil, J. Chapman, K. S. Novoselov, A. Mishchenko, Electrical and optical characterization of atomically thin ws2, Dalton transactions 43 (27) (2014) 10388-10391.

[61] J. Wilson, A. Yoffe, The transition metal dichalcogenides discussion and interpretation of the observed optical, electrical and structural properties, Advances in Physics 18 (73) (1969) 193-335.

[62] H. Zhong, G. Yang, H. Song, Q. Liao, H. Cui, P. Shen, C.-X. Wang, Vertically aligned graphene-like sns2 ultrathin nanosheet arrays: excellent energy storage, catalysis, photoconduction, and field-emitting performances, The Journal of Physical Chemistry C 116 (16) (2012) 9319-9326.

[63] C. R. Dean, A. F. Young, I. Meric, C. Lee, L. Wang, S. Sorgenfrei, K. Watanabe, T. Taniguchi, P. Kim, K. L. Shepard, et al., Boron nitride substrates for high-quality graphene electronics, Nature nanotechnology 5 (10) (2010) 722-726.

[64] W. Jin, P.-C. Yeh, N. Zaki, D. Chenet, G. Arefe, Y. Hao, A. Sala, T. O. Mentes, J. I. Dadap, A. Locatelli, et al., Tuning the electronic structure of monolayer graphene/mos2 van der waals heterostructures via interlayer twist, Physical Review B 92 (20) (2015) 201409. 